\title{
1 Targeted nanocarriers coopting pulmonary leukocytes for drug delivery to the
} injured brain

Patrick M. Glassman ${ }^{1, *}$, Jia Nong ${ }^{1, *}$, Jacob W. Myerson ${ }^{1}$, Viviana Zuluaga-Ramirez ${ }^{2}$, Alba Rodriguez-Garcia ${ }^{3,4}$, Alvin Mukalel ${ }^{5}$, Serena Omo-Lamai ${ }^{6}$, Landis R. Walsh ${ }^{1}$, Raisa Y. Kiseleva ${ }^{1}$, Carlos H. Villa ${ }^{1}$, Colin F. Greineder ${ }^{1}$, Scott E. Kasner ${ }^{7}$, Drew Weissman ${ }^{8}$, Michael J. Mitchell $^{5,9,10,11,12}$, Silvia Muro ${ }^{13,14,15}$, Yuri Persidsky ${ }^{2,16}$, Jacob S. Brenner ${ }^{1,6}$, Vladimir R. Muzykantov ${ }^{1, \#}$, Oscar A. Marcos-Contreras ${ }^{1,7, *, \#}$

${ }^{1}$ Department of Systems Pharmacology and Translational Therapeutics, Perelman School of Medicine, University of Pennsylvania, Philadelphia, PA, 19104, USA

${ }^{2}$ Department of Pathology and Laboratory Medicine, Lewis Katz School of Medicine, Temple University, Philadelphia, PA, 19140, USA

${ }^{3}$ Department of Pathology and Laboratory Medicine, Ovarian Cancer Research Center, Perelman School of Medicine, University of Pennsylvania, Philadelphia, PA, 19104, USA

${ }^{4}$ Center for Cellular Immunotherapies, Abramson Cancer Center, Perelman School of Medicine, University of Pennsylvania, Philadelphia, PA, 19104, USA

${ }^{5}$ Department of Bioengineering, University of Pennsylvania, Philadelphia, PA, 19104, USA

${ }^{6}$ Division of Pulmonary Allergy, and Critical Care, Department of Medicine, Perelman School of Medicine, University of Pennsylvania, Philadelphia, PA, 19104, USA

${ }^{7}$ Department of Neurology, Perelman School of Medicine, University of Pennsylvania, Philadelphia, PA, 19104, USA

${ }^{8}$ Division of Infectious Diseases, Perelman School of Medicine, University of Pennsylvania, Philadelphia, PA 19104, USA

${ }^{9}$ Abramson Cancer Center, Perelman School of Medicine, University of Pennsylvania, Philadelphia, PA, 19104, USA

${ }^{10}$ Institute for Immunology, Perelman School of Medicine, University of Pennsylvania, Philadelphia, PA, 19104, USA

${ }^{11}$ Cardiovascular Institute, Perelman School of Medicine, University of Pennsylvania, Philadelphia, PA, 19104, USA

${ }^{12}$ Institute for Regenerative Medicine, Perelman School of Medicine, University of Pennsylvania, Philadelphia, PA, 19104, USA

${ }^{13}$ Institute for Bioengineering of Catalonia (IBEC), Barcelona, Spain

${ }^{14}$ Institute of Catalonia for Research and Advanced Studies (ICREA), Barcelona, Spain

${ }^{15}$ Institute for Bioscience and Biotechnology (IBBR), College Park, MD, USA

${ }^{16}$ Center for Substance Abuse Research, Lewis Katz School of Medicine, Temple University, Philadelphia, PA, 19140, USA

* Denotes equal contribution.

\# Denotes corresponding author

To whom correspondence may be addressed. Email: oscarmar@pennmedicine.upenn.edu or muzykant@pennmedicine.upenn.edu 


\section{ABSTRACT}

Selective drug delivery to injured regions of the brain is an elusive, but biomedically important, goal. It is tempting to co-opt migrating white blood cells (WBC) to carry drugs to the injured brain, using natural WBC tropism. Current approaches to load cargoes to WBC have limited utility, particularly in acute conditions, due to the need for time consuming ex vivo manipulation and loading of cells. Physiological, in vivo loading of WBC may be advantageous in this scenario. Here we devised such a strategy, capitalizing on the unique features of the direct blood exchange between brain and lungs. Mediators emanating from the injured brain directly travel to the pulmonary vasculature via venous flow. In response to these mediators, WBCs, transiently residing in the pulmonary microvascular lumen, disembark and flow with arterial blood to the brain microvasculature, where they adhere and transmigrate to the brain parenchyma via the local chemoattractant gradient. We posited that direct in vivo targeting of cargoes to the pulmonary WBC pool may provide drug transfer to brain via this natural mechanism. To test this, we intravenously injected agents targeted to intercellular adhesion molecule 1 (ICAM) in mice with acute brain inflammation caused by direct injection of tumor necrosis factor alpha (TNF- $\alpha$ ). We found that: A) At 2 hours, $>20 \%$ of ICAM/NP accumulated in lungs, predominantly in WBCs; B) At 6 and 22 hours, ICAM/NP pulmonary uptake markedly decreased; C) In contrast, ICAM/NP uptake in brain increased $~ 5$-fold in this time interval, concomitantly with migration of WBCs to the brain. Cranial window fluorescent microscopy confirmed WBC transport of ICAM/NP to the brain in TNF- $\alpha$-challenged mice beyond the BBB. Importantly, demonstrating the pharmacologic relevance of this strategy, dexamethasoneloaded ICAM/liposomes abrogated brain edema in this model. In sum, coopting the natural homing of WBC from the lungs via ICAM-targeting to injured brain is an attractive strategy for precise interventions for treatment of acute brain injuries.

\section{VISUAL ABSTRACT}

\section{Pathophysiology}

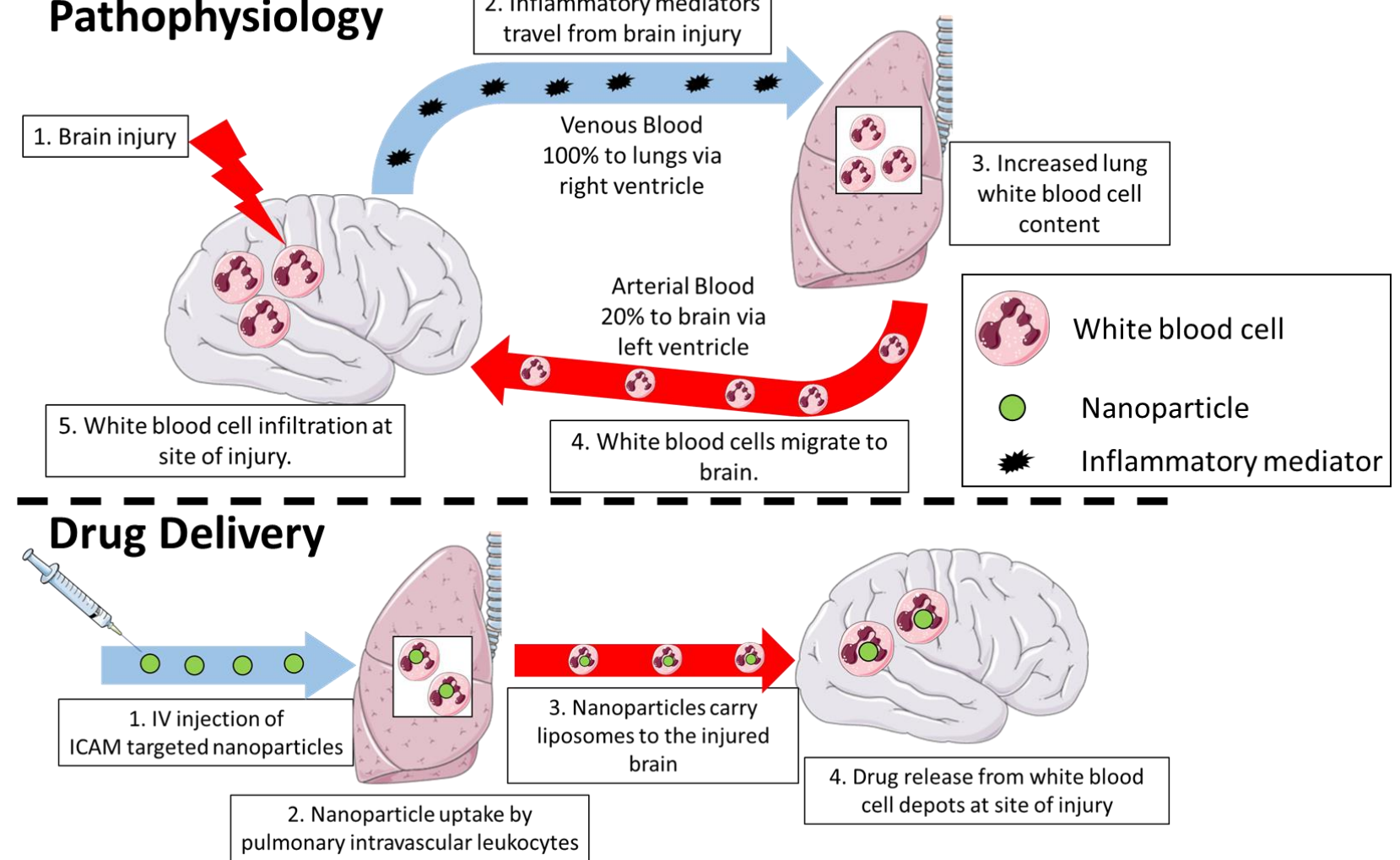




\section{INTRODUCTION}

Targeted drug delivery to the brain promises breakthroughs in treatment of debilitating and lethal pathologies, including stroke, traumatic brain injury, glioblastoma and other brain tumors, meningitis, and neurodegenerative diseases ${ }^{1,2}$. Various carriers with distinct chemistry, geometry, mechanical flexibility, and affinity have been devised to achieve this elusive goal, ${ }^{3,4}$, 6,7 . One approach to enhance delivery employs targeting to and across the cerebral vasculature using antibodies, peptides, and other ligands of molecules that are stably expressed on the luminal surface of brain vessels. However, targeting to these molecules, including receptors for transferrin, insulin, and growth factors does not provide selectivity for sites of injury and inflammation ${ }^{8,9}$. In order to achieve enhanced specificity for injured regions of the brain, targeting to inducible cell adhesion molecules (CAMs) expressed on endothelial cells, such as vascular CAM (VCAM) ${ }^{9}$, has been tested and has shown improved delivery and pharmacologic effects. Despite these inroads, direct, specific delivery to the parenchyma of the injured region of the brain remains an elusive goal.

To achieve the formidable goal of delivering drugs to the brain parenchyma within injured areas of the brain, it is tempting to utilize the natural homing of leukocytes to the pathologically altered region of the brain ${ }^{10,11,12,13}$. This natural tropism is mediated by several processes including: A) activation of endothelial cells and white blood cells (WBC) by inflammatory mediators, B) increased exposure of adhesion molecules on both endothelial cells and WBC, C) disruption of endothelial tight junctions ${ }^{14,15}$, and D) attraction of circulating leukocytes via a chemokine gradient emanating from the site of injury ${ }^{16}$. The idea of using isolated, ex vivo loaded WBC is attractive and several groups have reported therapeutic benefits of injecting drug-loaded WBCs in animal models of neurological disorders ${ }^{17,18,19,20,21,22}$. Data on the fate of injected WBC (clearance, distribution, and effects on the body) are largely unknown. This aspect of the brain drug delivery requires systematic direct tracing of isotope-labeled agents ${ }^{23}$. However, a priori, this strategy is only permissive of loading a small number of cells and ex vivo manipulation may lead to undesired activation or alteration of these cells, resulting in severe adverse effects ${ }^{24,25,26 \text {, }}$ 27. Additionally, ex vivo manipulation of cells is not suitable for acute, emergency conditions and would require a specialized facility (e.g. as in CAR-T therapy) that is not likely to be found at most hospitals. A more desirable approach would be to specifically load those WBC that are predisposed to localize to the injured brain with drugs or drug carriers in vivo, bypassing the need for any ex vivo manipulation. It has been reported that ICAM is expressed on the surface of many WBCs, including monocytes and neutrophils ${ }^{28}$. Following inflammatory stimuli, the surface expression of ICAM on immune cells is significantly upregulated ${ }^{29,30,31,32}$, providing selectivity for delivery to activated WBCs by targeting to ICAM.

We postulate the ideal cells for this role are pulmonary intravascular leukocytes. The pulmonary circulation hosts the largest and most dynamic pool of intravascular WBC that are poised to quickly respond to local and remote signals from damaged tissues ${ }^{33,} 34$. There are no intervening capillary beds between the directly interconnected cerebral and pulmonary vasculatures. Hence, the constituent cells of the pulmonary vasculature (endothelial, pulmonary intravascular leukocytes) is the first set of extra-cerebral cells receiving signals from inflammatory mediators emanating from brain injuries (e.g. cytokines, exosomes, damageassociated molecular patterns). In fact, distal injuries often induce a secondary pulmonary pathology $35,36,37,38$, which results in an increase in and hyperactivation of the pulmonary WBC pool. There are several reports that host defense cells responding to chronic neurological disorders mature in the lungs prior to trafficking to the injured brain ${ }^{39,40,41}$. These considerations imply that the dynamic pool of pulmonary WBCs are ideally positioned to shuttle 
drugs directly from the lungs to sites of brain pathology. However, strategies for controllable, specific, effective, and safe loading of drugs into intravascular WBCs have not been reported.

In the current study, we characterized the dynamic localization of leukocytes and ICAM-targeted pharmacological agents in the blood, lungs, and brain in a murine model of acute neurovascular inflammation induced by direct injection of tumor necrosis factor alpha (TNF- $\alpha$ ) into the brain parenchyma. Our data presented below indicate that in the lungs, the number of WBC, the uptake of ICAM-targeted agents, and their fraction taken by intravascular WBC rapidly increased to the peak at 2 hours, followed by profound decline by $24 \mathrm{~h}$. In the brain, in contrast, these cells gradually accumulated and increased $\sim 5$-fold 24 hours after TNF- $\alpha$ injury. Intravital fluorescent microscopy showed that in mice challenged with cranial injection of TNF, the migrating ICAM/NP-carrying WBCs accumulated in the brain parenchyma.

Leukocyte-mediated delivery to the brain parenchmya originating from ICAM-targeted lung uptake was demonstrated in the present study for monoclonal antibodies (mAb) and for three types of nanoparticles: polystyrene nanoparticles, liposomes, and lipid nanoparticles (LNP). IV injection of ICAM-targeted liposomes loaded with dexamethasone completely abrogated brain edema induced by TNF- $\alpha$. These results indicate: A) Direct, in vivo leukocyte loading: after IV injection in mice with acute neurovascular inflammation, ICAM-targeted nanoparticles rapidly bind to pulmonary leukocytes in vivo; B) Natural leukocyte trafficking: these loaded leukocytes traffic to the inflamed region of the brain; and C) Leukocyte-mediated drug delivery to the parenchyma: this approach enables shuttling of nanoparticles to the site of brain injury, ultimately resulting in therapeutic efficacy. Overall, direct, in vivo targeting of the pulmonary WBC pool shortly after brain injury provides a mechanism to harness this dynamic pool of cells for selective drug delivery to the brain.

\section{RESULTS}

A systemic response to acute neurovascular inflammation in mouse model of intracranial TNF- $\alpha$ injection: In order to assess changes in accessible ICAM following brain injection of TNF-a, the tissue uptake of anti-ICAM ( $\alpha$ ICAM) mAb was investigated at several time points post injury. The direct quantitative measurements using isotope-labeled agents showed that: A) there was no significant differences in blood concentrations at different time points (Figure 1a, Supplemental Table 1); B) lung uptake reached a peak 2 hours post-injury and declined to baseline levels by 6 hours post-injury, suggesting a transient increase in ICAM levels in lungs post-brain injury (Figure 1b); C) brain uptake increased progressively with time after TNF- $\alpha$ insult, with aICAM brain delivery increasing 7-fold over naïve levels at 24 hours after injury (Figure 1c). Similar experiments were carried out for aCD45, which behaved with identical dynamics as aICAM, with a 4.6-fold increase in lung delivery 2 hours post-injury and a 16-fold increase in brain delivery 24 hours after injury. (Figure 1d, e, f).

Experiments were performed to evaluate dynamics of immune cells in blood, lungs, and brain following brain injury. Complete blood counts (CBC) revealed that the TNF- $\alpha$ injury affected circulating immune cells in several ways: A) transient increases in circulating neutrophils and monocytes, peaking 6 hours post-TNF- $\alpha$; and B) a transient decrease in circulating lymphocytes, reaching a nadir at the same time point (Figure 1g). Flow cytometry evaluated cell type distributions in the lungs 2 hours after TNF and in the brain 24 hours after TNF, i.e., at the post-injury time points with the maximal level of aICAM uptake in the two corresponding organs. This analysis revealed that comparing with basal levels measured in naïve mice, at 2 hours post-injury, there was a significant increase in lung leukocytes (Figure 1h). 24 hours post-injury, there was a 14-fold increase in the relative recovery of brain leukocytes (Figure 1i). 

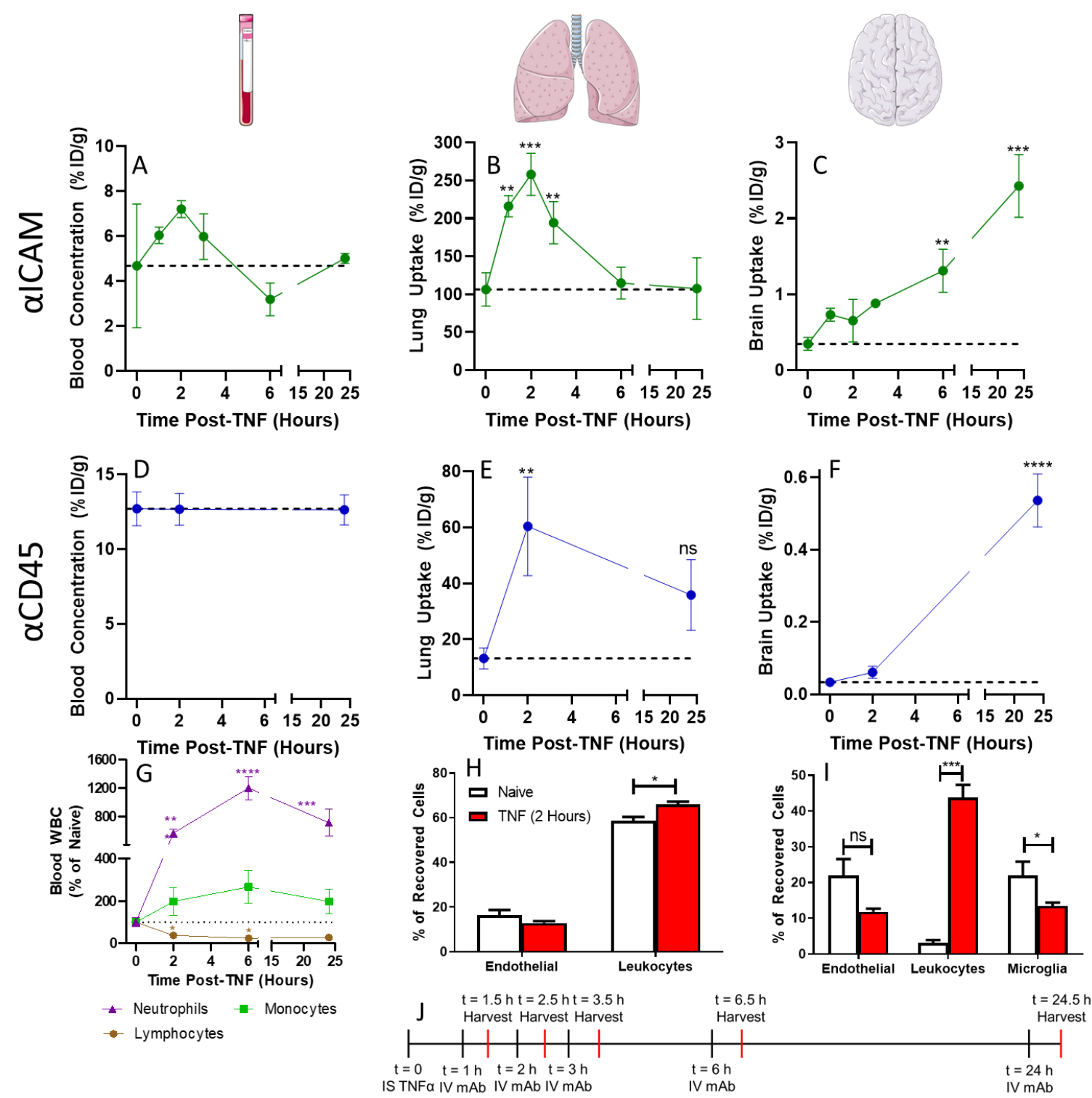

176

Figure 1. Local injection of TNF- $\alpha$ in the brain induces a systemic response. Following IV injection of alCAM, A) blood, B) lung, and C) brain targeting was assessed at several time points post-TNF. Similar studies were performed for aCD45 biodistribution in D) blood, E) lungs, and F) brain. Data represented as percent of injected dose per gram tissue (\%ID/g). G) Complete blood counts were used to measure dynamic changes of white blood cells in circulation following TNF- $\alpha$. Flow cytometry of single cell suspensions obtained from $\mathrm{H}$ ) lungs 2 hours post-TNF- $\alpha$ and I) brain 24 hours post-TNF- $\alpha$. Endothelial cells: CD $31^{+}$CD 45 , Leukocytes: $\mathrm{CD}^{+} 5^{+}$, Microglia: $\mathrm{CD} 45^{\text {mid }}$. J) Timeline of biodistribution experiments. Data represented as mean \pm SEM. Dashed lines represent levels in naïve mice. Comparisons in A-G made by 1-way ANOVA with Dunnett's post-hoc test vs. naïve mice and comparisons in $\mathrm{H}-\mathrm{I}$ made by unpaired t-test. $\mathrm{N}=3$ /group.

These results are consistent with the following hypothetical spatiotemporal characteristics of the bi-directional vascular transport between the brain and lungs, illustrated in figure 2a. Proinflammatory mediators emanating from the site of brain injury are transported by blood pumped via the right heart chambers directly to the lungs collecting $100 \%$ of the venous blood ejected by 
the right ventricle. The mediators activate endothelial and white blood cells in the pulmonary vasculature, and ensuing interaction of these cellular constituencies further attract and activate circulating WBC to the lung microvasculature, serving as a transient "training base", from which primed WBCs get transferred to the target organ passively with arterial blood flow (note: brain takes disproportionally high $15-20 \%$ of the cardiac arterial blood output), adhere to pathologically activated cerebral endothelial cells and transmigrate to the injured parenchyma. Furthermore, our results indicate that targeting to ICAM enables loading to the pulmonary WBCs permitting the subsequent trip to the brain just described above.

201

202

203

204

205

206

207

208

209

210

211

212

213

214

215

216

217

218

219

220

221

222

223

224

225

226

227

ICAM-targeted monoclonal antibodies (mAbs) migrate to the brain: Encouraged by the identification of a lung-brain axis following brain injury (Figure 2a), we performed studies to appraise the utility of this novel drug delivery paradigm. Here, we injected isotope-labeled affinity ligands including aICAM into mice 2 hours post-TNF-a injury to evaluate the role of target epitope/cell type on pharmacokinetics and biodistribution (Figure $\mathbf{2 b}$ ).

aPECAM behaved as expected for ligands of epitopes constitutively and stably on the surface of endothelial cells showing: A) specific (vs. IgG, see below) uptake in most organs at early time points; B) decreasing tissue concentrations over time (Figure 2c, Supplemental Table 2); and, C) more rapid blood clearance vs. control IgG (Supplemental Figure 1). In part due to more prolonged circulation time, control IgG slowly accumulated in the brain due enhanced vascular permeability, which has been previously reported in this model $^{9}$ (Supplemental Table 2, Supplemental Figure 2).

The PK/BD of aICAM was more complex and rather unanticipated in some aspects. Over time, lung concentrations of aICAM decreased with a simultaneous increase in brain uptake aICAM (Figure 2d, Supplemental Table 2). The distribution pattern of aCD45 was similar to that of alCAM, with specific accumulation in lungs at early time points, followed by lung clearance and slow delivery to the brain (Figure 2e, Supplemental Table 2). Because CD45 is a panleukocyte marker, its accumulation can be attributed to an influx of mAb-tagged leukocytes at the injury in the brain. There was a significant correlation between clearance from the lung and changes in brain uptake with time (Supplemental Figure 3). It was hypothesized that this unexpected distribution pattern of aICAM was due to initial delivery of aICAM to activated leukocytes in the pulmonary vasculature followed by migration of leukocytes to the injured brain. 

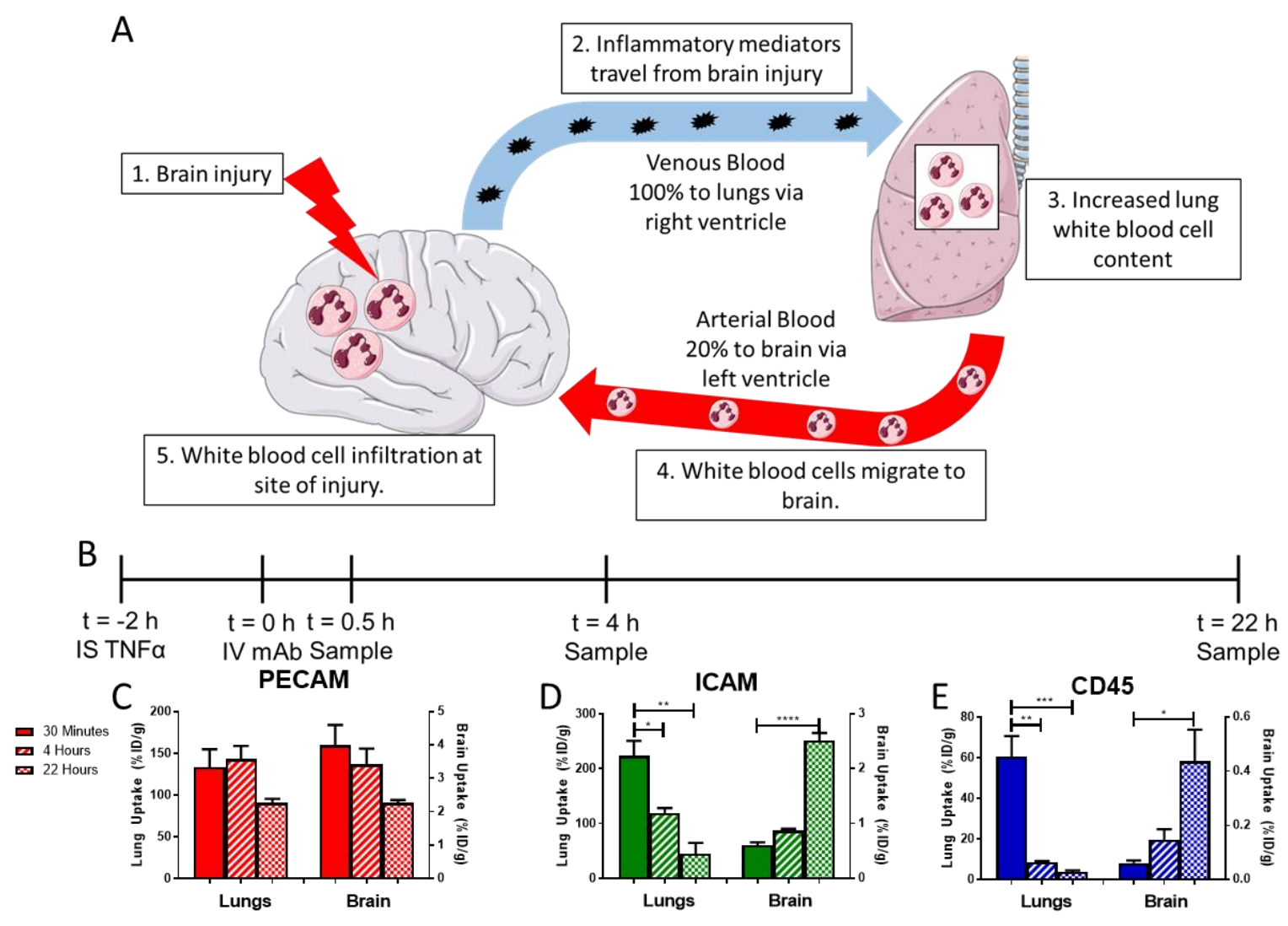

Figure 2. aICAM and $\alpha \mathrm{CD} 45 \mathrm{mAbs}$ accumulate in the lungs, then migrate to the brain. A) Schematic of proposed mechanism underlying leukocyte migration. B) PK study timeline. Lung and brain pharmacokinetics of mAbs directed against: c) PECAM, D) ICAM, and E) CD45 following IV injection 2 hours post-TNF- $\alpha$ injury. Time points reflect the time post-mAb injection when organs were harvested. Data represented as mean \pm SEM. Comparisons made by 1 -way ANOVA with Dunnett's post-hoc test vs. 30 minutes. $\mathrm{N}=3$ /group.

Diversification of ICAM-directed loading of nanoparticles to lung WBC for subsequent delivery to the brain: For this purpose, we compared three different types of ICAM-targeted nanoparticles: polystyrene nanoparticles, liposomes, and LNP (Figure 3a). ICAM-targeted nanoparticles were largely cleared from the blood within 30 minutes; however, there was a rebound in blood concentrations over the next several hours for ICAM-targeted nanoparticles, potentially reflecting redistribution of leukocytes carrying nanoparticles into blood (Supplemental Figure 4). Similar to aICAM mAb, ICAM-targeted nanoparticles were largely taken up in the lungs within 30 minutes of injection (polystyrene nanoparticles: $147 \pm 1 \% \mathrm{ID} / \mathrm{g}$, liposome: $174 \pm 6$ $\%$ ID/g, LNP: $123 \pm 9 \% \mathrm{ID} / \mathrm{g}$ ), followed by clearance from the lungs over several hours (Figure 3b, c, d, Supplemental Tables 3, 4, 5). Both polystyrene nanoparticles and LNP displayed monotonic increases in brain concentrations with time after injection, while liposomes had a transient increase in brain uptake (Figure 3b, c, d, Supplemental Table 4). To evaluate the interplay between lung clearance and brain uptake of nanoparticles, lung/brain ratios were calculated at time points post-dose. All three particles displayed a steady increase in this ratio with time, reflecting the opposite trends in tissue targeting kinetics (Figure $\mathbf{3 e , ~ f , ~ g , ~}$ Supplemental Tables 3, 4, 5). On the contrary, untargeted control IgG nanoparticles did not display significant accumulation in either lungs or brain (concentrations $>10$-fold lower than ICAM-targeted) (Supplemental Figure 5, Supplemental Tables 3, 4, 5). 
A
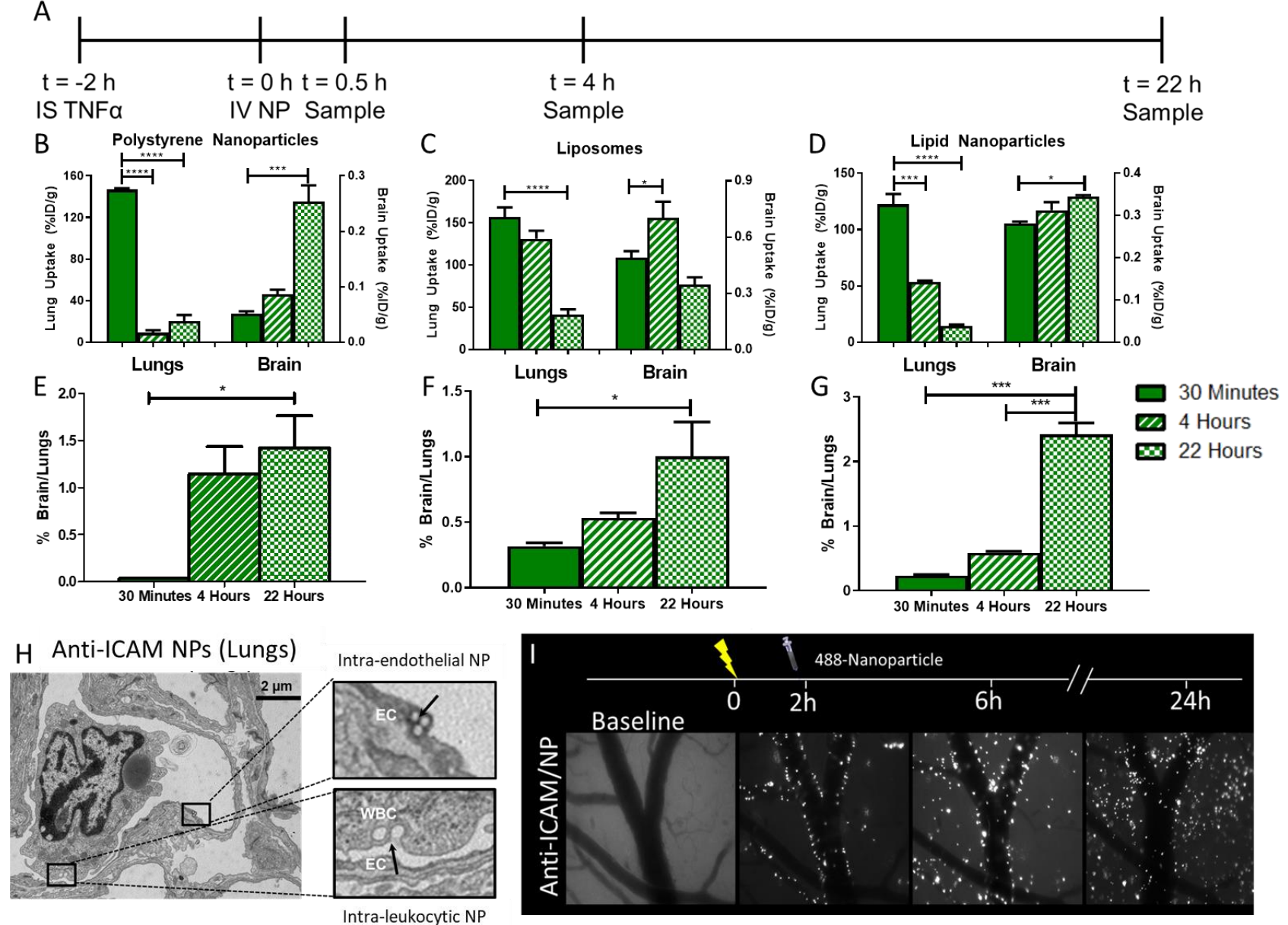

258

259

260

261

262

263

264

265

266

267

268

269

270

271

272

273

274

275

276

Figure 3: ICAM-targeted nanoparticles accumulate in the inflamed brain. A) Study timeline. Pharmacokinetics of B) polystyrene nanoparticles, C) liposomes, and D) lipid nanoparticles in lungs and brain following injection. Kinetic changes in the ratio of nanoparticles in brain vs. lungs for E) polystyrene nanoparticles, F) liposomes, and G) lipid nanoparticles. H) Transmission electron microscopy of ICAM-targeted polystyrene nanoparticles in lung endothelium and leukocytes 30 minutes post-injection. I) Cranial window intravital microscopy of ICAM-targeted polystyrene nanoparticles in TNF- $\alpha$ injured brain. Data represented as mean \pm SEM. Comparisons in B, C, D were made by 1-way ANOVA with Dunnett's post-hoc test vs. 30 minutes and those in $E, F, G$ were made by 1-way ANOVA with Tukey's post-hoc test. $N \geq$ 3/group.

Additional studies focused on visualizing the delivery mechanisms of ICAM-targeted nanoparticles in both lungs and brain. Transmission electron microscopy (TEM) demonstrated ICAM-targeted polystyrene nanoparticle localization to both endothelial cells and leukocytes in the lungs 30 minutes after IV injection (Figure $3 \mathbf{h}$ ). Cranial window intravital microscopy (Figure 3i) showed; A) ICAM-targeted polystyrene nanoparticles were associated with the walls of inflamed brain blood vessels immediately following IV injection; B) consistent with radiotracing experiments, the number of nanoparticles in the cranial window increased over time after 
injection; C) 4 hours after injection, nanoparticles appeared in clusters and some beads were detected in the parenchyma; D) 22 hours after injection, nanoparticle fluorescence was no longer confined to large vessel walls and had spread into the parenchyma, suggesting that ICAM-targeted nanoparticles access a mechanism to cross the blood-brain barrier. Similar data were obtained for ICAM-targeted liposomes using cranial window intravital microscopy, with liposome fluorescence lining the vessel walls immediately post-injection and gradually accumulating in the brain parenchyma over 22 hours (Supplemental Figure 6). The fluorescent signal for liposomes was more diffuse than that for polystyrene nanoparticles, possibly reflecting differences in particle stability following internalization.

ICAM targeted nanoparticles are predominantly delivered to leukocytes: Single cell suspensions were prepared from lungs 30 minutes after injection of ICAM-targeted nanoparticles (2 hours post TNF- $\alpha$ injury) (Figure 4a). Flow cytometry analysis showed that nearly all nanoparticlepositive cells in the lungs were leukocytes $\left(C D 45^{+}\right)(93.4 \pm 1.4 \%$ of recovered cells), with the remaining NC-positive cells being identified as endothelial cells $\left(\mathrm{CD} 31^{+}\right)$(Figure 4b).

292

293

294

295

296

297

298

Having identified leukocytes as the primary target cells for ICAM-targeted nanoparticles in the lungs of TNF- $\alpha$-challenged mice, we tested the hypothesis that these mobile leukocytes deliver alCAM/nanoparticles to the inflamed brain 22 hours after nanoparticle injection (24 hours postinjury). In single cell suspensions prepared from the brain, essentially all nanoparticle-positive cells were leukocytes ( $98.7 \pm 0.2 \%$ of recovered cells) (Figure 4c). Flow cytometry showed polystyrene nanoparticle uptake in the brain for pristine and non-specific lgG-coated polystyrene nanoparticles, agreeing with biodistribution data (Supplemental Figure 7). A sub-typing of cells in the brain revealed that the majority of nanoparticle-positive leukocytes in the brain were monocytes/macrophages $(73.0 \pm 9.7 \%)$, with the bulk of the remainder being neutrophils $(24.5 \pm$ 9.9\%) (Supplemental Figure 8 and 9). A large fraction of monocytes/macrophages were nanoparticle-positive $(40.5 \pm 3.6 \%)$. Among other leukocytes, $27.4 \pm 6.6 \%$ of neutrophils and $25.2 \pm 1.2 \%$ of other myeloid cells were nanoparticle-positive. Minimal ICAM-targeted nanoparticle uptake in microglia and T-cells (Supplemental Figure 9b).

Brain histology confirmed nanoparticle association with macrophages (CD68-stained) (Figure 4d, Supplemental Figure 10a) and endothelial cells (VCAM-stained) (Figure 4e, Supplemental Figure 10b). Histology indicated greater uptake of ICAM-targeted nanoparticles vs. IgG-coated nanoparticles, both in the vasculature and in the brain parenchyma (Figure 4f, g, Supplemental Figure 10a, b). Parenchymal nanoparticle fluorescence was largely colocalized with macrophages, consistent with flow cytometry results. 


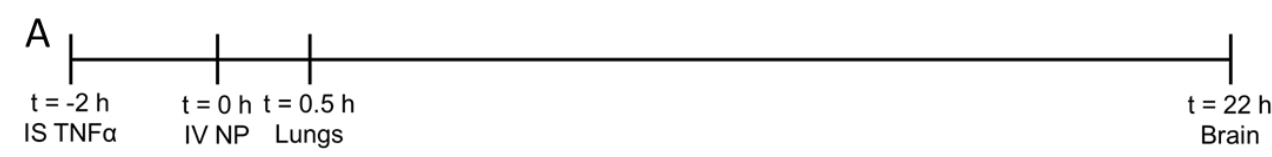

B Distribution of NP to Lung Cell Types

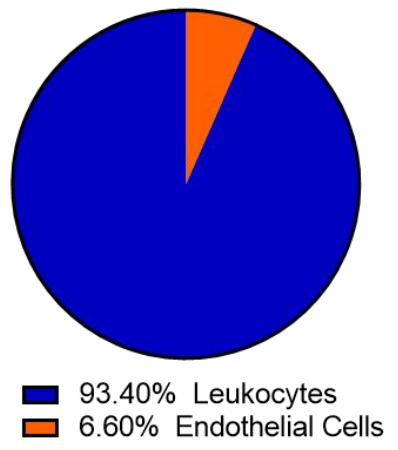

Distribution of NP to Brain Cell Types

C

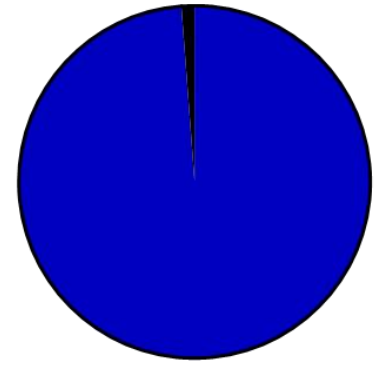

- $98.80 \%$ Leukocytes

- $1.20 \%$ Double Negative
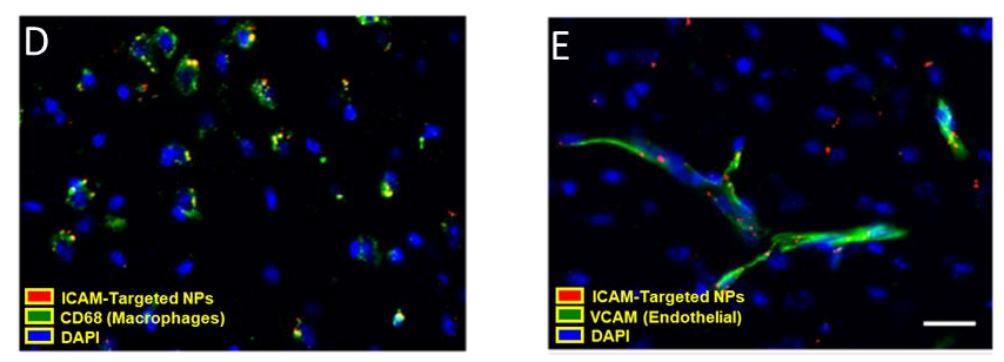

Figure 4: Cellular specificity of ICAM-targeted polystyrene nanoparticles. A) Flow cytometry was performed on single cell suspensions obtained from lungs and brain at the designated times post-nanoparticle injection. The fraction of nanoparticles recovered in B) lungs and $\mathrm{C}$ ) brain that were associated with specific cell types. Leukocytes: CD45 ${ }^{+}$, Endothelium: CD $31^{+} \mathrm{CD} 45^{-}$. Histology of brain tissue sections collected 22 hours post-injection of polystyrene nanoparticles in TNF- $\alpha$ challenged mice. Nanoparticle association with macrophages (CD68 ${ }^{+}$) and endothelial cells $\left(\mathrm{VCAM}^{+}\right)$was measured for $\left.\mathrm{D}, \mathrm{E}\right)$ ICAM-targeted and $\left.\mathrm{F}, \mathrm{G}\right) \lg \mathrm{G}$ nanoparticles. Scale bar: $50 \mu \mathrm{m}$. Data represented as mean \pm SEM. $N=3 /$ group.

Drug loaded ICAM-targeted liposomes reduce brain edema: Brain injection of TNF-a leads to reproducible brain edema, as assessed by measuring extravasation of radiolabeled albumin. Liposomes were loaded with dexamethasone (Supplemental Table 6 and Supplemental Figure 11) and free dexamethasone, dexamethasone-loaded IgG liposomes, and dexamethasone-loaded ICAM-targeted liposomes were assessed for effects on brain edema (Figure 5a, Supplemental Figure 12). No significant effects were detected for IV injection of $0.5 \mathrm{mg} / \mathrm{kg}$ free dexamethasone $(-0.531 \pm 26.3 \%$ protection) or dexamethasone-loaded $\lg \mathrm{G}$ liposomes with equivalent drug dose $(24.3 \pm 18.9 \%$ protection). Dexamethasone-loaded ICAMtargeted liposomes provided near complete protection from edema (88.5 $\pm 14.6 \%$ protection) 
(Figure 5b). Precluding effects of the liposomes themselves, neither empty IgG liposomes (40.7 $\pm 23.5 \%$ protection) nor ICAM-targeted liposomes $(-4.14 \pm 29.81 \%$ protection) provided significant protection against edema (Figure 5b).

Complete blood counts were performed to assess the impact of dexamethasone loaded into ICAM-targeted liposomes and other formulations on blood cells (Supplemental Figure 13). ICAM-targeted dexamethasone liposomes led to a reduction in lymphocytes, consistent with the known mechanism of action of the drug, but no other blood cell parameters were affected by treatment, indicating that the therapeutic effect of ICAM-targeted liposomal dexamethasone represents localized action in the brain rather than a systemic effect.

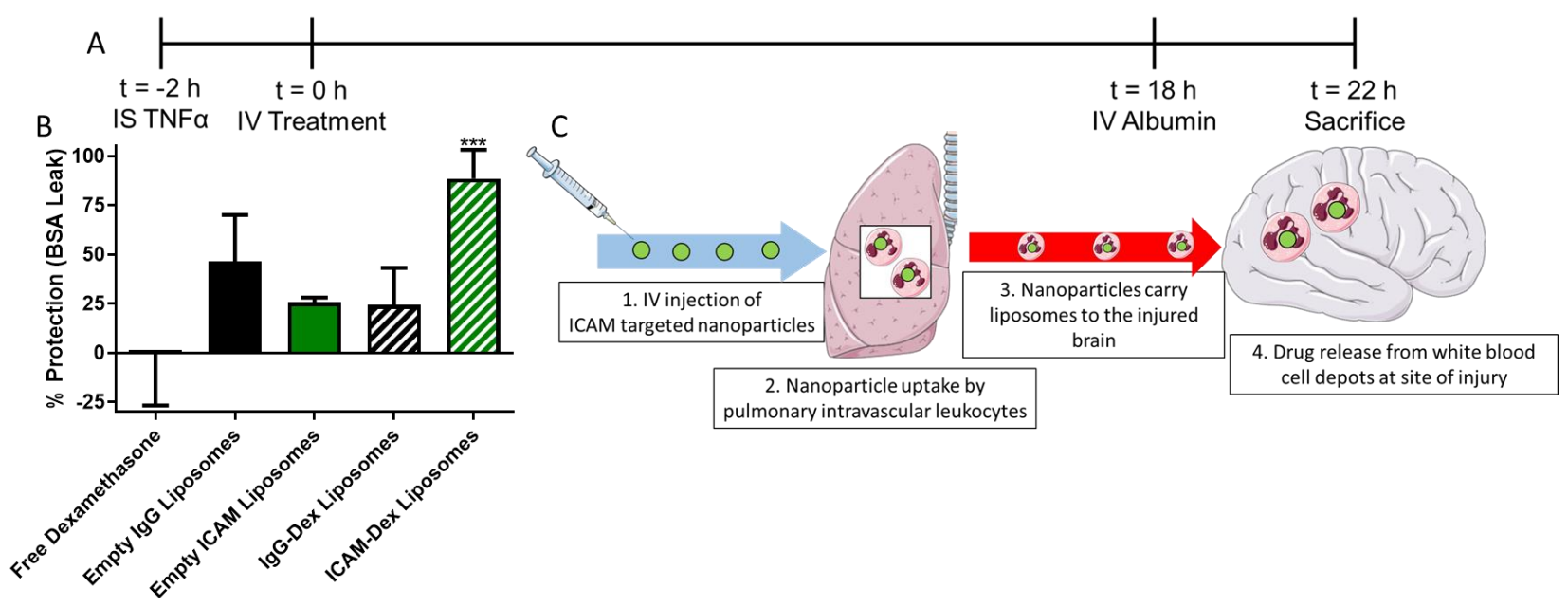

Figure 5. ICAM-targeted dexamethasone (Dex) liposomes protect mice from TNF-induced brain edema. A) Experimental timeline. B) Protective effects of ICAM-targeted dexamethasone liposomes $(0.5 \mathrm{mg} / \mathrm{kg}$ dexamethasone). As controls for Dex-loaded liposomes, equivalent doses of empty IgG or ICAM-targeted liposomes were tested. \% protection was calculated assuming $100 \%$ protection as equivalent to edema induced by sham injury and $0 \%$ protection as equivalent to edema induced by TNF injury without treatment (Supplemental Figure 12). C) Proposed model of leukocyte-mediated drug delivery. Data displayed as mean \pm SEM. Comparisons made by 1 -way ANOVA with Dunnett's post-hoc test vs. untreated (solid line, $0 \%$ protection). $\mathrm{N} \geq 3$ /group.

\section{DISCUSSION}

Development of effective therapies for neurological disorders presents formidable challenges including limited success in targeted drug delivery to the brain and especially into the required components of the parenchyma - neurons, glia, etc. The pressing need for effective targeted therapies is especially aggravated in patients suffering from acute brain injuries including stroke, traumatic brain injury, neuroinflammation, and intracranial hemorrhage. These patients present additional challenges for the pharmacotherapy including but not limited to complicating factors, including: 1) rapid disease progression, 2) multiple pathophysiological factors, and 3) poor tolerance for adverse effects.

Harnessing natural host defense mechanisms by loading nanoparticles into leukocytes responding to signals emanating from the injured brain is an attractive strategy for drug delivery. In this case, delivery to sites of injury would be controlled by the natural homing mechanisms used by leukocytes to reach the brain (e.g. emanating chemokine gradients, cell adhesion 
molecules, etc.). Leukocytes have been used as carriers in chronic neurodegenerative conditions following ex vivo loading of drugs and reinfusion into animals ${ }^{21,42,43}$. In these studies, it was suggested that leukocytes (or leukocyte-derived extracellular vesicles) could not only reach the brain, but also mediate transfer of their cargo into neurons in order to elicit a pharmacologic response. ${ }^{44,45}$. Through direct targeting of leukocytes in vivo, the need for complex ex vivo manipulations can be bypassed in a manner that is permissive for selective delivery into the brain parenchyma

We postulated that direct targeting of pulmonary intravascular leukocytes would be a viable strategy to achieve selective drug delivery to injured regions of the brain, which has several potential advantages vs. ex vivo modification, including: 1) treatment can be initiated rapidly after injury, without the need for ex vivo modification of cells, 2) all leukocytes accessible to IV injected $\mathrm{mAb} /$ nanoparticles are potential targets for loading, 3) selection for specific leukocyte phenotypes is possible by targeting to specific markers, and 4) leukocytes could be converted into drug depots/biofactories that concentrate drugs in the inflamed region where their activity is required. By targeting ICAM expressed on the surface of activated leukocytes, a decline in lung concentrations was seen in parallel with delivery to the injured brain.

Following IV administration, affinity ligands directed towards many vascular epitopes have low levels of delivery to organs such as the brain, in part due to first pass binding to the pulmonary endothelium. However, by directly targeting cell populations that transiently reside in the lungs (e.g. intravascular leukocytes), conversion of the lung from a competitor into an active participant in delivery to the brain is feasible. The data presented above show that targeting to molecules expressed on all (CD45) and activated (ICAM) leukocytes permits delivery to the brain, despite significant uptake by the lungs. The purported mechanism for this delivery is that ICAM-targeted nanoparticles rapidly bind to pulmonary intravascular leukocytes and remain associated with leukocytes, likely in an intracellular compartment following CAM-mediated endocytosis ${ }^{46}$, as they migrate to the brain in response to inflammatory signaling.

Following studies aimed at suggesting a mechanism of delivery to the brain, we pursued therapeutic studies to elucidate the therapeutic relevance of this leukocyte-based drug delivery strategy. We selected the small molecule corticosteroid dexamethasone as a therapeutic agent. Notably, dexamethasone has been tested in clinical trials for treatment of acute ischemic stroke, but ultimately failed due to off-target effects. Among its pleiotropic effects, dexamethasone downregulates expression of the following: inducible CAMs, inflammatory cytokine expression (IL-1, IL-6, TNF- $\alpha$ ), cyclooxygenase-2, collagenase, and NF-KB ${ }^{47}$. We hypothesized that ICAMtargeted dexamethasone-loaded liposomes would provide selective delivery of dexamethasone to the injured brain. The results presented here demonstrate that IV injection of dexamethasone 2 hours post-TNF injury was only able to prevent brain edema when encapsulated in ICAMtargeted liposomes (Figure 5b). These results are likely due to not only changes in local brain concentrations of dexamethasone, but also due to direct effects on leukocytes targeted by this strategy.

In summary, we have developed a novel approach for direct, in vivo loading of activated leukocytes with nanoparticles via targeting to ICAM-1 (Figure 5c). We propose the following mechanism for brain delivery whereby the pulmonary intravascular leukocytes: 1) respond to signals emanating from the injured brain and change their activation status and local concentration, 2) are targeted by aICAM mAbs and nanoparticles, and 3) shuttle the taken up alCAM mAb/nanoparticles from the lungs to the injured region of the brain. Our results show that direct leukocyte targeting provides a steady accumulation of nanoparticles into the brain parenchyma following induction of acute neurovascular inflammation. Essentially all of the 
targeted nanoparticles in the brain were associated with leukocytes, namely monocytes/macrophages and neutrophils. Injection of ICAM-targeted, dexamethasone-loaded liposomes into mice two hours post-TNF injury was able to completely protect mice from injuryinduced brain edema. By harnessing natural leukocyte migration patterns, this strategy provides enhanced selectivity for the injured region of the brain and has potential for applications in other acute neurovascular inflammatory injuries such as stroke.

\section{MATERIALS AND METHODS}

Reagents: Reagents for iodination of proteins were obtained from the following sources: $\mathrm{Na}^{125}$ I (PerkinElmer, Waltham, MA), 1,3,4,6-tetrachloro-3a,6a-diphenyl-glycouril (lodogen ${ }^{\circledR}$ ) (Pierce, Rockford, IL). Polystyrene beads $(190 \mathrm{~nm})$ were purchased from Bangslabs (Fishers, IN). All lipids for liposome formulation were obtained from Avanti Polar Lipids (Alabaster, AL). Pooled rat $\lg G$ (rlgG) was purchased from Invitrogen (Carlsbad, CA). All other chemicals and reagents were purchased from SigmaAldrich (St. Louis, MO), unless specifically noted.

Animals: All animal studies were carried out in accordance with the Guide for the Care and Use of Laboratory Animals (National Institutes of Health, Bethesda, MD) and all animal protocols were approved by the University of Pennsylvania Institutional Animal Care and Use Committee. All animal experiments were carried out using male, 6-8 week old C57BL/6 mice (20-25 g) (The Jackson Laboratory, Bar Harbor, ME).

Protein Production and Purification: Anti-ICAM mAb (YN1) was produced and purified from hybridoma supernatants, as described previously ${ }^{48}$. Purification of YN1 was performed using Protein $\mathrm{G}$ affinity chromatography.

Radiolabeling: Antibodies (YN1, rlgG) were radiolabeled with ${ }^{125}$ I via the lodogen ${ }^{\circledR}$ method. Briefly, tubes were coated with $100 \mu \mathrm{g}$ of lodogen ${ }^{\circledR}$ reagent were incubated with antibodies (1-2 $\mathrm{mg} / \mathrm{mL})$ and $\mathrm{Na}^{125} \mathrm{I}(0.25 \mu \mathrm{Ci} / \mu \mathrm{g}$ protein) for 5 minutes on ice. Residual free iodine was removed from the bulk solution using a desalting column and thin layer chromatography was used to confirm the efficiency of radiolabeling. As a quality control step, all proteins were confirmed to have $<10 \%$ free ${ }^{125}$ I prior to further use.

Polystyrene Nanoparticle Conjugation: Carboxylated, polystyrene beads were conjugated to antibodies (rlgG, YN1) via reaction of N-hydroxysulfosuccinimide (sulfo-NHS) $(0.275 \mathrm{mg} / \mathrm{mL})$, 1 ethyl-3-(3-dimethylaminopropl)carbodiimide $\mathrm{HCl}$ (EDC) $(0.1 \mathrm{mg} / \mathrm{mL})$, and 200 antibody molecules/bead. For experiments involving radioisotope tracing, 15\% of the total antibody added to the reaction was ${ }^{125}$ I-labeled rlgG. NC size and polydispersity index (PDI) were confirmed via dynamic light scattering (DLS).

Liposome Formulation: Liposomes were prepared as described previously ${ }^{48}$. Briefly, 1,2dipalmitoyl-sn-glycero-3-phosphocholine (DPPC), cholesterol, and 1,2-distearoyl-sn-glycero-3phosphoethanolamine-N-[azido(polyethyleneglycol)-2000 (DSPE-PEG2000-azide) were mixed in a molar ratio of 54:40:6. Liposomes were prepared via the thin film extrusion method. To form drug loaded liposomes, the lipid film was hydrated in a solution containing $20 \mathrm{mg} / \mathrm{mL}$ of dexamethasone-21-phosphate in phosphate buffered saline (PBS), pH 7.4. The resulting vesicles were extruded through $200 \mathrm{~nm}$ polycarbonate membranes.

Lipid Nanoparticle (LNP) Formulation: LNPs were prepared via microfluidic mixing as previously described $^{49}$. Briefly, an ethanol phase was prepared by combining ionizable lipid, 1,2-dioleoylsn-glycero-3-phosphoethanolamine (DOPE), cholesterol, 1,2-dimyristoyl-sn-glycero-3- 
phosphoethanolamine- $N$-[methoxy(polyethylene glycol)-2000] (C14-PEG2000) at molar ratios of 35:16:46.5:2.5, respectively. Separately, an aqueous phase was prepared by resuspending scrambled siRNA sequences in $10 \mathrm{mM}$ citrate buffer to a concentration of $.75 \mathrm{mg} / \mathrm{mL}$. Ethanol and aqueous phases were then mixed in a single channel microfluidic device at a 3:1 ratio using a syringe pump ${ }^{50}$. LNPs were dialyzed against $1 x$ PBS for 2 hours at room temperature, followed by sterile filtration using $.22 \mu \mathrm{m}$ syringe filters.

Conjugation of antibodies to the liposome surface was carried out using strain-promoted alkyneazide cycloaddition. Antibodies were functionalized by reacting with a 5-fold molar excess of dibenzocyclooctyne-PEG ${ }_{4}$-NHS ester (DBCO-PEG4-NHS) (Click Chemistry Tools, Scottsdale, AZ) for 30 minutes at room temperature. Unreacted DBCO-PEG4-NHS was removed via centrifugation through a molecular weight cutoff (MWCO) filter. Liposomes were conjugated with DBCO-functionalized antibodies by reacting for 4 hours at $37^{\circ} \mathrm{C}$. For experiments involving radiotracing, $10 \%$ of the total antibody added was ${ }^{125} \mathrm{I}$-labeled $\mathrm{rlgG}$. Unconjugated antibody was removed from the liposomes using gel filtration chromatography. The size, distribution, and concentration of liposomes was determined using DLS and nanoparticle tracking analysis (Malvern Panalytical, Westborough, MA).

Dexamethasone Loading and Release: Both the amount of dexamethasone loading into liposomes and kinetics of release were assessed using reverse phase high performance liquid chromatography (HPLC). The mobile phase consisted of $30 \% \mathrm{v} / \mathrm{v}$ acetonitrile, $70 \% \mathrm{v} / \mathrm{v}$ water, and $0.1 \% \mathrm{v} / \mathrm{v}$ trifluoroacetic acid. Buffer was run at a flow rate of $0.6 \mathrm{~mL} /$ minute through a $\mathrm{C} 8$ column (Exlipse XDB-C8, $3 \mu \mathrm{m}, 3.0 \times 100 \mathrm{~mm}$, Phenomenex). Dexamethasone was detected using UV absorbance at $240 \mathrm{~nm}$ and the assay had a linear range of $1.56-100 \mu \mathrm{g} / \mathrm{mL}$

(Supplemental Figure 14). Drug release was measured by dialyzing loaded particles against a large excess of PBS, $\mathrm{pH} 7.4$ at $37^{\circ} \mathrm{C}$ and collecting samples at designated time points.

TNF Injury Model: Neurovascular inflammation was induced in mice via a unilateral injection of TNF- $\alpha(0.5 \mu \mathrm{g} / \mathrm{mouse}, 2.5 \mu \mathrm{L}$, BioLegend) into the striatum using a stereotaxic frame at the following coordinates relative to the bregma: $0.5 \mathrm{~mm}$ anterior, $2.0 \mathrm{~mm}$ lateral, $-3 \mathrm{~mm}$ ventral ${ }^{10}$. At different times relative to TNF- $\alpha$ injection (1-24 hours), mice were injected intravenously with a bolus dose of either mAbs $(5 \mu \mathrm{g})$ or nanoparticles (polystyrene beads, liposomes). Animals were perfused with $20 \mathrm{~mL}$ of PBS, $\mathrm{pH} 7.4$ prior to collecting organs for further analysis. For pharmacokinetic and biodistribution studies, the amount of radioactivity in blood and organs was measured using a gamma counter (Wizard2, PerkinElmer, Waltham, MA).

Transmission Electron Microscopy: Visualization of NC uptake in the lungs shortly after injection was performed using TEM, as previously described ${ }^{51}$. Briefly, 30 minutes post-injection, lungs were fixed with $2.5 \%$ glutaraldehyde and $4 \%$ paraformaldehyde in $0.1 \mathrm{M}$ sodium cacodylate buffer, then processed into $80-90 \mathrm{~nm}$-thin resin-embedded sections to visualization by TEM.

Intravital Microscopy: After removing the meninges, a cranial window was opened in one parietal bone of mice. This window was sealed with a glass coverslip and a cannula (PlasticsOne, Roanoke, VA) was placed into the subarachnoid space adjacent to the window (1 $\mathrm{mm}$ depth). Animals were allowed to recover for 5 days between opening of the cranial window and injection of TNF- $\alpha$ to prevent any artifacts related to surgery-induced inflammation. In vivo imaging was performed in real time with a Stereo Discovery V20 fluorescence microscope (Carl Zeiss AG, Oberkochen, Germany).

Flow Cytometry: Single cell suspensions of brain were produced as described previously ${ }^{9,52}$. Briefly, tissues were enzymatically digested with dispase and collagenase for 1 hour at $37^{\circ} \mathrm{C}$, 
followed by addition of $600 \mathrm{U} / \mathrm{mL}$ DNase Grade II. Tissue digests were demyelinated in Percoll and ACK buffer (Quality Biological, Gaithersburg, MD) was added to lyse any residual RBCs. Samples were then filtered through: 1) $100 \mu \mathrm{m}$ nylon strainers and 2) $70 \mu \mathrm{m}$ nylon strainers (ThermoFisher).

Cells were then stained with appropriate antibodies (Supplemental Table 7). Briefly, $2 \times 10^{6}$ cells were labeleld per tube in PBS containing $2 \% \mathrm{v} / \mathrm{v}$ fetal bovine serum (FBS). Fc receptors were blocked using TruStain FcX PLUS (anti-mouse CD16/32, 1:200 dilution) (BioLegend). In pilot experiments to determine localization of $\mathrm{NC}$ in leukocytes $\left(\mathrm{CD} 45^{+}\right)$vs. endothelial cells $\left(\mathrm{CD} 31^{+}\right)$, flow cytometry was performed using an Accuri C6plus (Benton Dickinson, San Jose, $\mathrm{CA}$ ). Detailed subtyping of white blood cells in the brain was performed using the strategy described by Posel et al. using a BD LSRFortessa (Benton Dickinson, San Jose, CA) flow cytometer. Live/dead staining was performed using LIVE/DEAD Fixable Aqua Dead Cell Stain Kit (1:1000 dilution, ThermoFisher). In this assay uptake by the following cell types was defined: 1) microglia $\left(C D 45^{\text {mid }}\right)$, T-cells $\left(C D 45^{\text {hi }} C D 3^{+}\right)$, neutrophils $\left(C D 45^{\text {hi }}\right.$ Ly $\left._{6 G} G^{+}\right)$, monocytes/macrophages (CD45 hi CD3 Ly6G ${ }^{-}$CD11 $\left.\mathrm{b}^{+} \mathrm{Ly}^{-} \mathrm{C}^{+}\right)$. Analysis of flow cytometry data was performed using the BD Accuri C6 software (Benton Dickinson, San Jose, CA) and FlowJo v10.6.2 (Tree Star).

Histology: TNF brains injected with IgG- or aICAM conjugated NCs were perfused, harvested 24 hours-post injected, and fixed in $4 \%$ paraformaldehyde. After freezing in tissue freezing medium, the brains were sectioned at $20 \mu \mathrm{m}$ thickness. Tissue sections were then permeabilized and blocked in blocking solution ( $5 \%$ normal goat serum and $0.3 \%$ Triton X-100 in PBS) for 1 hour at room temperature, then incubated overnight at $4{ }^{\circ} \mathrm{C}$ with primary antibodies (Supplemental Table 8) in blocking solution. After washing with PBS, the sections were incubated with secondary antibodies conjugated with Alexa fluorophores (1:200, Invitrogen) in PBS for 1 hour at room temperature. After washing, the sections were counterstained with nuclei dye 4'-6Diamidino-2-phenylindole (DAPI, Southern, Biotech). The images were taken by Leica DM6000 Widefield Microscope.

Therapeutic Studies: The effects of dexamethasone on TNF-induced brain edema were assessed as described in our previous publication ${ }^{9}$. Briefly, 2 hours post-TNF injection, mice were dosed IV with either: 1) $0.5 \mathrm{mg} / \mathrm{kg}$ dexamethasone, 2) empty liposomes (either aICAM or IgG coated), or 3) $0.5 \mathrm{mg} / \mathrm{kg}$ liposomal dexamethasone (either alCAM or IgG coated). 20 hours after TNF injection, mice were injected with ${ }^{125}$ I-labeled bovine serum albumin (BSA, $~ 3 \times 10^{6}$ $\mathrm{cpm} /$ mouse), which was then allowed to circulate for 4 hours. After BSA circulation, mice were perfused with $20 \mathrm{~mL}$ of PBS, pH 7.4, over 5 minutes and organs were harvested. Edema was determined by measuring the relative concentration of extravasated BSA in the brain to the concentration in the bloodstream. For calculations of therapeutic efficacy, $0 \%$ protection was defined using PBS-treated, TNF-injured mice and 100\% protection was defined using PBStreated, sham-injured mice.

Complete Blood Counts: At designated time points, blood was collected from mice into tubes containing EDTA. Blood cells were analyzed using an Abaxis VetScan HM5 Hematology Analyzer and all values were normalized to the mean value obtained for naïve mice.

Statistics: All statistical tests were performed using GraphPad Prism 8 (GraphPad Software, San Diego, CA). ${ }^{*}$ denotes $p<0.05,{ }^{* *}$ denotes $p<0.01,{ }^{* * *}$ denotes $p<0.001,{ }^{* * * *}$ denotes $\mathrm{p}<0.0001$. 
574

575

576

577

578

579

580

581

582

583

584

585

586

587

588

589

590

591

592

593

594

595

596

597

598

599

600

601

602

603

604

605

606

607

608

609

610

611

612

613

614

615

\section{Funding Sources}

O.A.M.C. received support from the American Heart Association (Grant 19CDA345900001). V.R.M and J.S.B. received support from the Cardiovascular Institute of the University of Pennsylvania. V.R.M received funding from the National Institutes of Health $(\mathrm{NIH})(\mathrm{R} 01$ HL155106, R01 HL128398, R01 HL143806). P.M.G. received funding from the National Institutes of Health (K99 HL153696). M.J.M. acknowledges support from a US National Institutes of Health Director's New Innovator Award (DP2 TR002776), a Burroughs Wellcome Fund Career Award at the Scientific Interface (CASI), a grant from the American Cancer Society (129784-IRG-16-188-38-IRG), and the National Institutes of Health (NCI R01 CA241661, NCl R37 CA244911, and NIDDK R01 DK123049).

\section{REFERENCES}

1. Patel MM, Goyal BR, Bhadada SV, Bhatt JS, Amin AF. Getting into the brain: approaches to enhance brain drug delivery. CNS Drugs 2009, 23(1): 35-58.

2. Finbloom JA, Sousa F, Stevens MM, Desai TA. Engineering the drug carrier biointerface to overcome biological barriers to drug delivery. Adv Drug Deliv Rev 2020, 167: 89-108.

3. Chen EM, Quijano AR, Seo YE, Jackson C, Josowitz AD, Noorbakhsh S, et al. Biodegradable PEGpoly(omega-pentadecalactone-co-p-dioxanone) nanoparticles for enhanced and sustained drug delivery to treat brain tumors. Biomaterials 2018, 178: 193-203.

4. Song E, Gaudin A, King AR, Seo YE, Suh HW, Deng Y, et al. Surface chemistry governs cellular tropism of nanoparticles in the brain. Nat Commun 2017, 8: 15322.

5. Yoo D, Magsam AW, Kelly AM, Stayton PS, Kievit FM, Convertine AJ. Core-Cross-Linked Nanoparticles Reduce Neuroinflammation and Improve Outcome in a Mouse Model of Traumatic Brain Injury. ACS Nano 2017, 11(9): 8600-8611.

6. Alghamri MS, McClellan BL, Hartlage MS, Haase S, Faisal SM, Thalla R, et al. Targeting Neuroinflammation in Brain Cancer: Uncovering Mechanisms, Pharmacological Targets, and Neuropharmaceutical Developments. Front Pharmacol 2021, 12: 680021.

7. Altshuler DB, Kadiyala P, Nunez FJ, Nunez FM, Carney S, Alghamri MS, et al. Prospects of biological and synthetic pharmacotherapies for glioblastoma. Expert Opin Biol Ther 2020, 20(3): 305-317.

8. Marcos-Contreras OA, Brenner JS, Kiseleva RY, Zuluaga-Ramirez V, Greineder CF, Villa CH, et al. Combining vascular targeting and the local first pass provides 100-fold higher uptake of ICAM-1targeted vs untargeted nanocarriers in the inflamed brain. J Control Release 2019, 301: 54-61. 
632

633

634

635

636

637

638

639

640

641

642

643

644

645

646

647

648

649

650

651

652

653

654

655

9. Marcos-Contreras OA, Greineder CF, Kiseleva RY, Parhiz H, Walsh LR, Zuluaga-Ramirez V, et al. Selective targeting of nanomedicine to inflamed cerebral vasculature to enhance the bloodbrain barrier. Proc Natl Acad Sci U S A 2020, 117(7): 3405-3414.

10. DirnagI U, ladecola C, Moskowitz MA. Pathobiology of ischaemic stroke: an integrated view. Trends Neurosci 1999, 22(9): 391-397.

11. Abdul-Muneer PM, Chandra N, Haorah J. Interactions of oxidative stress and neurovascular inflammation in the pathogenesis of traumatic brain injury. Mol Neurobiol 2015, 51(3): 966-979.

12. Tohidpour A, Morgun AV, Boitsova EB, Malinovskaya NA, Martynova GP, Khilazheva ED, et al. Neuroinflammation and Infection: Molecular Mechanisms Associated with Dysfunction of Neurovascular Unit. Front Cell Infect Microbiol 2017, 7: 276.

13. Naveed M, Zhou QG, Han F. Cerebrovascular inflammation: A critical trigger for neurovascular injury? Neurochem Int 2019, 126: 165-177.

14. Ma Q, Chen S, Klebe D, Zhang JH, Tang J. Adhesion molecules in CNS disorders: biomarker and therapeutic targets. CNS Neurol Disord Drug Targets 2013, 12(3): 392-404.

15. Frijns CJ, Kappelle LJ. Inflammatory cell adhesion molecules in ischemic cerebrovascular disease. Stroke 2002, 33(8): 2115-2122.

16. Ahmad M, Graham SH. Inflammation after stroke: mechanisms and therapeutic approaches. Trans/ Stroke Res 2010, 1(2): 74-84.

17. Batrakova EV, Li S, Reynolds AD, Mosley RL, Bronich TK, Kabanov AV, et al. A macrophagenanozyme delivery system for Parkinson's disease. Bioconjug Chem 2007, 18(5): 1498-1506.

18. Zhao Y, Haney MJ, Mahajan V, Reiner BC, Dunaevsky A, Mosley RL, et al. Active Targeted Macrophage-mediated Delivery of Catalase to Affected Brain Regions in Models of Parkinson's Disease. J Nanomed Nanotechnol 2011, S4.

19. Dou H, Grotepas CB, McMillan JM, Destache CJ, Chaubal M, Werling J, et al. Macrophage delivery of nanoformulated antiretroviral drug to the brain in a murine model of neuroAIDS. $J$ Immunol 2009, 183(1): 661-669.

20. Pang L, Qin J, Han L, Zhao W, Liang J, Xie Z, et al. Exploiting macrophages as targeted carrier to guide nanoparticles into glioma. Oncotarget 2016, 7(24): 37081-37091. 
21. Anselmo AC, Gilbert JB, Kumar S, Gupta V, Cohen RE, Rubner MF, et al. Monocyte-mediated delivery of polymeric backpacks to inflamed tissues: a generalized strategy to deliver drugs to treat inflammation. J Control Release 2015, 199: 29-36.

22. Chavas TEJ, Su FY, Srinivasan S, Roy D, Lee B, Lovelace-Macon L, et al. A macrophage-targeted platform for extending drug dosing with polymer prodrugs for pulmonary infection prophylaxis. J Control Release 2021, 330: 284-292.

23. Deng H, Konopka CJ, Cross TL, Swanson KS, Dobrucki LW, Smith AM. Multimodal Nanocarrier Probes Reveal Superior Biodistribution Quantification by Isotopic Analysis over Fluorescence. ACS Nano 2020, 14(1): 509-523.

24. Kim JY, Park J, Chang JY, Kim SH, Lee JE. Inflammation after Ischemic Stroke: The Role of Leukocytes and Glial Cells. Exp Neurobiol 2016, 25(5): 241-251.

25. Arvin B, Neville LF, Barone FC, Feuerstein GZ. The role of inflammation and cytokines in brain injury. Neurosci Biobehav Rev 1996, 20(3): 445-452.

26. Xue M, Yong VW. Matrix metalloproteinases in intracerebral hemorrhage. Neurol Res 2008, 30(8): 775-782.

27. Hartl R, Schurer L, Schmid-Schonbein GW, del Zoppo GJ. Experimental antileukocyte interventions in cerebral ischemia. J Cereb Blood Flow Metab 1996, 16(6): 1108-1119.

28. Bui TM, Wiesolek HL, Sumagin R. ICAM-1: A master regulator of cellular responses in inflammation, injury resolution, and tumorigenesis. J Leukoc Biol 2020, 108(3): 787-799.

29. Elsner J, Sach M, Knopf HP, Norgauer J, Kapp A, Schollmeyer P, et al. Synthesis and surface expression of ICAM-1 in polymorphonuclear neutrophilic leukocytes in normal subjects and during inflammatory disease. Immunobiology 1995, 193(5): 456-464.

30. Hubbard AK, Rothlein R. Intercellular adhesion molecule-1 (ICAM-1) expression and cell signaling cascades. Free Radic Biol Med 2000, 28(9): 1379-1386.

31. Real E, Kaiser A, Raposo G, Amara A, Nardin A, Trautmann A, et al. Immature dendritic cells (DCs) use chemokines and intercellular adhesion molecule (ICAM)-1, but not DC-specific ICAM-3grabbing nonintegrin, to stimulate CD4+ T cells in the absence of exogenous antigen. $J$ Immunol 2004, 173(1): 50-60. 
32. Wiesolek HL, Bui TM, Lee JJ, Dalal P, Finkielsztein A, Batra A, et al. Intercellular Adhesion Molecule 1 Functions as an Efferocytosis Receptor in Inflammatory Macrophages. Am J Pathol 2020, 190(4): 874-885.

33. Rosales C, Demaurex N, Lowell CA, Uribe-Querol E. Neutrophils: Their Role in Innate and Adaptive Immunity. J Immunol Res 2016, 2016: 1469780.

34. Eliseeva SI, Knowlden ZA, Lester GM, Dean DA, Georas SN, Chapman TJ. Changes in lung immune cell infiltrates after electric field treatment in mice. Sci Rep 2021, 11(1): 1453.

35. Kalsotra A, Zhao J, Anakk S, Dash PK, Strobel HW. Brain trauma leads to enhanced lung inflammation and injury: evidence for role of P4504Fs in resolution. J Cereb Blood Flow Metab 2007, 27(5): 963-974.

36. Koutsoukou A, Katsiari M, Orfanos SE, Kotanidou A, Daganou M, Kyriakopoulou M, et al. Respiratory mechanics in brain injury: A review. World J Crit Care Med 2016, 5(1): 65-73.

37. Hu PJ, Pittet JF, Kerby JD, Bosarge PL, Wagener BM. Acute brain trauma, lung injury, and pneumonia: more than just altered mental status and decreased airway protection. Am J Physiol Lung Cell Mol Physiol 2017, 313(1): L1-L15.

38. Samary CS, Ramos AB, Maia LA, Rocha NN, Santos CL, Magalhaes RF, et al. Focal ischemic stroke leads to lung injury and reduces alveolar macrophage phagocytic capability in rats. Crit Care 2018, 22(1): 249.

39. Kuehl C, Thati S, Sullivan B, Sestak J, Thompson M, Siahaan T, et al. Pulmonary Administration of Soluble Antigen Arrays Is Superior to Antigen in Treatment of Experimental Autoimmune Encephalomyelitis. J Pharm Sci 2017, 106(11): 3293-3302.

40. Odoardi F, Sie C, Streyl K, Ulaganathan VK, Schlager C, Lodygin D, et al. T cells become licensed in the lung to enter the central nervous system. Nature 2012, 488(7413): 675-679.

41. Saito E, Gurczynski SJ, Kramer KR, Wilke CA, Miller SD, Moore BB, et al. Modulating lung immune cells by pulmonary delivery of antigen-specific nanoparticles to treat autoimmune disease. $S c i$ Adv 2020, 6(42).

42. Klyachko NL, Polak R, Haney MJ, Zhao Y, Gomes Neto RJ, Hill MC, et al. Macrophages with cellular backpacks for targeted drug delivery to the brain. Biomaterials 2017, 140: 79-87. 
43. Zhang C, Ling CL, Pang L, Wang Q, Liu JX, Wang BS, et al. Direct Macromolecular Drug Delivery to Cerebral Ischemia Area using Neutrophil-Mediated Nanoparticles. Theranostics 2017, 7(13): 3260-3275.

44. Haney MJ, Zhao Y, Harrison EB, Mahajan V, Ahmed S, He Z, et al. Specific transfection of inflamed brain by macrophages: a new therapeutic strategy for neurodegenerative diseases. PLoS One 2013, 8(4): e61852.

45. Zhao Y, Haney MJ, Jin YS, Uvarov O, Vinod N, Lee YZ, et al. GDNF-expressing macrophages restore motor functions at a severe late-stage, and produce long-term neuroprotective effects at an early-stage of Parkinson's disease in transgenic Parkin Q311X(A) mice. J Control Release 2019, 315: 139-149.

46. Muro S, Wiewrodt R, Thomas A, Koniaris L, Albelda SM, Muzykantov VR, et al. A novel endocytic pathway induced by clustering endothelial ICAM-1 or PECAM-1. J Cell Sci 2003, 116(Pt 8): 15991609.

47. P. B, W. SJ. Adrenocorticotropic Hormone, Adrenal Steroids, and the Adrenal Cortex. Goodman \& Gilman's: The Pharmacological Basis of Therapeutics, 13 edn, 2018.

48. Hood ED, Greineder CF, Shuvaeva T, Walsh L, Villa CH, Muzykantov VR. Vascular Targeting of Radiolabeled Liposomes with Bio-Orthogonally Conjugated Ligands: Single Chain Fragments Provide Higher Specificity than Antibodies. Bioconjug Chem 2018, 29(11): 3626-3637.

49. Billingsley MM, Singh N, Ravikumar P, Zhang R, June CH, Mitchell MJ. Ionizable Lipid Nanoparticle-Mediated mRNA Delivery for Human CAR T Cell Engineering. Nano Lett 2020, 20(3): 1578-1589.

50. Shepherd SJ, Warzecha CC, Yadavali S, El-Mayta R, Alameh MG, Wang L, et al. Scalable mRNA and siRNA Lipid Nanoparticle Production Using a Parallelized Microfluidic Device. Nano Lett 2021, 21(13): 5671-5680.

51. Garnacho C, Dhami R, Solomon M, Schuchman EH, Muro S. Enhanced Delivery and Effects of Acid Sphingomyelinase by ICAM-1-Targeted Nanocarriers in Type B Niemann-Pick Disease Mice. Mol Ther 2017, 25(7): 1686-1696.

52. Posel C, Moller K, Boltze J, Wagner DC, Weise G. Isolation and Flow Cytometric Analysis of Immune Cells from the Ischemic Mouse Brain. J Vis Exp 2016(108): 53658. 